\title{
Efecto historeparador de un extracto proteico y vitamínico en Allium cepa L. con daño cromosómico
}

\section{Historeparative effect of a protein and vitamin extract in Allium cepa $\mathbf{L}$. with chromosomal damage}

\author{
Luis Felipe Gonzales Llontop ${ }^{1}$, Mariel del Rocío Chotón Calvo ${ }^{2}$, Julio Chico Ruiz ${ }^{3}$
}

\section{RESUMEN}

El objetivo fue evaluar el efecto historeparador de un extracto proteico y vitamínico (tónico INTI) en Allium cepa L. "cebolla" con daño cromosómico inducido por penicilina benzatínica. Se escogió radículas de $3,0 \mathrm{~cm}$ de longitud de 30 bulbos de Allium cepa L. Se empleó un diseño experimental con 5 tratamientos, señalando las raicillas cada 10 minutos para tomar a las células en diversos momentos de mitosis. Luego se sometieron a la técnica de coloración de Tjio y Levan. Las raicillas de A. cepa L. tratados con penicilina benzatínica 1\% presentaron trastornos cromosómicos de tipo: puente $(11,3 \%)$, fragmentaciones $(16,7 \%)$, otros trastornos $(38,3 \%)$ y sin trastornos $(33,4 \%)$; mientras el grupo que se administró la penicilina benzatínica $1 \%$ más el extracto exhibió: puente $(2,1 \%)$, fragmentaciones $(7,2 \%)$, otros trastornos $(10,4 \%)$ y sin trastornos $(80,3 \%)$. El extracto ensayado posee efecto reparador al reducir proporcionalmente las aberraciones cromosómicas descubiertas en células de Allium cepa tratadas con penicilina benzatínica $1 \%$. En las condiciones de laboratorio empleadas a una dosis de 10 $\mathrm{mL}$ del extracto utilizado se comprobó el notable efecto historeparador.

Palabras clave: Efecto citoreparador, trastornos cromosómicos.

\begin{abstract}
The objective was to evaluate the historeparative effect of a protein and vitamin extract (INTI tonic) in Allium cepa L. "onion" with chromosomal damage induced by benzathine penicillin. $3.0 \mathrm{~cm}$ long radicles were chosen from 30 bulbs of Allium cepa L. An experimental design with 5 treatments was used, marking the roots every 10 minutes to take the cells at various times of mitosis. They then underwent Tjio and Levan's coloring technique. Roots of A. cepa L. treated with $1 \%$ benzathine penicillin presented chromosomal disorders of the type: bridging (11.3\%), fragmentations $(16.7 \%)$, other disorders $(38.3 \%)$ and no disorders $(33,4 \%)$; while the group that was administered $1 \%$ benzathine penicillin plus the extract exhibited: bridging $(2.1 \%)$, fragmentations $(7.2 \%)$, other disorders $(10.4 \%)$ and without disorders $(80.3 \%)$. The extract tested has a repairing effect by proportionally reducing the chromosomal aberrations discovered in Allium cepa cells treated with $1 \%$ benzathine penicillin. In the laboratory conditions used at a dose of $10 \mathrm{~mL}$ of the extract used, the remarkable historeparator effect was verified.
\end{abstract}

Keywords: Cytoreparative effect, chromosomal disorders.

\footnotetext{
Biólogo. Docente asociado de la Facultad de Educación y Ciencias de la Comunicación de la Universidad Nacional Toribio Rodríguez de Mendoza de Amazonas. Doctor en Ciencias Biomédicas. Correo electrónico: luifego@hotmail.com

${ }^{2}$ Lic. en Estadística. Docente asociado de la Facultad de Educación y Ciencias de la Comunicación de la Universidad Nacional Toribio Rodríguez de Mendoza de Amazonas. Doctora en Gestión Pública y Gobernabilidad. Correo electrónico: marielchoton@hotmail.com

${ }^{3}$ Biólogo. Docente asociado de la Facultad de Ciencias Biológicas de la Universidad Nacional de Trujillo. Doctor en Ciencias Biológicas. Correo electrónico:j.chico@unitru.edu.pe
} 


\section{INTRODUCCIÓN}

En la era de la globalización, muchos agentes físicos, químicos y radioactivos presentes en el medio ambiente han incrementado la incidencia y aparición de enfermedades incurables que han incitado gran interés (Gonzáles, 2015; Kir et al., 2016; Causil et al., 2017). Es inmediato que una adecuada limitación de la exposición del hombre a sustancias toxicas y cancerígenas salvaría muchas vidas. Es urgente identificar que sustancias que pululan en el medio ambiente son potencialmente citotóxicas, entonces el ser humano informado deberá tomar sus medidas de precaución. En lo que se refiere a sustancias químicas inductoras de tejidos malignos no es una labor fácil.

La penicilina benzatínica es un antibiótico utilizado contra las enfermedades causadas por bacterias como la faringitis streptocócica, sífilis, difteria, fiebre reumática y otras; puede causar en el paciente reacciones adversas como alergias, dermatitis exfoliativa, erupción cutánea, urticaria, gastritis, reacciones gastrointestinales, dolor de cabeza, etc. Es menester buscar alternativas que a corto, mediano y a largo plazo que brinden al paciente la esperanza de curarse y no provocarle con el paso del tiempo una muerte lenta o rápida con el uso de fármacos y sus reacciones adversas (Katzung y Trevor, 2016).

A nivel mundial se calcula que más de 100 mil productos químicos diferentes elaborados por el hombre son de uso comercial e industrial corriente: cada año salen al mercado entre 500 y 1000 productos químicos nuevos como los fármacos destacando los bactericidas y los AINES (Rang et al., 2016; Gonzales et al., 2020).

El material génico (ADN) sea de una célula vegetal o animal, considerando los millones de células que conforman la estructura molecular de los seres vivos, son susceptibles a perjuicio tóxico corriendo el riesgo de ser alterados por agentes químicos y físicos (Oram, 2013). En el ámbito científico se usan modelos de citotoxicidad en plantas demostrando que se puede repotenciar la maquinaria de reparación celular usando extractos de plantas como la sábila, sangre de grado, etc. sobre el tejido perjudicado (Gonzales y Díaz, 2016; Alberts et al., 2014).

Varios de los fármacos consumidos por seres humanos inducen daños secundarios y ha sido demostrado usando como material biológico bacterias y animales de experimentación; sin embargo la acción regeneradora sobre tejidos afectados por parte el sustancias ricas en nutrientes (aminoácidos esenciales y vitaminas) no ha sido probada experimentalmente (Mamani, 2017).

El entendimiento y la comprensión del cáncer originado por agentes químicos se detuvo por el hecho de que el daño al ADN, aun siendo esencial para el inicio del tumor maligno, no incita por sí sola la transmutación cancerosa: se requieren otros factores adicionales para estimular la complejidad de procesos celulares que finiquitan la transformación maligna. Los elementos que lesionan al ADN son, por tanto, únicamente carcinógenos en potencia (Croce y Klein, 2013; Kuchy et al., 2016; Haq et al., 2017).

El tónico INTI (extracto proteico y vitáminico) como el aminol-extra (bioestimulante vegetal) son estimulantes biológicos a base de aminoácidos esenciales y vitaminas; siendo los aminoácidos compuestos primarios de la vida y constituyentes fundamentales de las proteínas, de extraordinaria asimilación por parte de los seres vivos. Se suministra directamente a los tejidos de los organismos eucariotas para su utilización estructural y fisiológica (Rang et al., 2016; Malca et al., 2020).

En este estudio nos propusimos evaluar el efecto historeparador de un extracto proteico y vitamínico (tónico INTI) en Allium cepa L. "cebolla" con daño cromosómico inducido por penicilina benzatínica.

\section{MATERIAL Y MÉTODO}

Adquisición de los bulbos de A. cepa L.: Los bulbos de Allium cepa L. "cebolla" variedad arequipeña fueron adquiridos en el mercado "Modelo" de la ciudad de Chachapoyas y clasificados taxonómicamente por un curator de la Universidad Nacional "Toribio Rodríguez de Mendoza" de Amazonas.

Diseño experimental: Se usó 30 bulbos de Allium сера L. El número de bulbos utilizado por tratamiento fue de 6 y se hicieron 5 tratamientos. Los bulbos fueron colocados en agua permanentemente restaurada con renovada aireación en oscuridad y a temperatura de $20^{\circ} \mathrm{C}$. Tres días después se seleccionaron solamente los ápices que tuvieron una longitud promedio de $3,0 \mathrm{~cm}$.

Logro de preparados celulares: Terminado el tratamiento de las raicillas, se cortaron los ápices de las raicillas para supeditarlas a la técnica de Tjio y Levan. Los preparados celulares fueron observados a 1000X en un microscopio binocular Leyca, donde se analizó la tabulación celular cada 1000 campos microscópicos. La genotoxicidad se evaluó mediante la observación de la clase y número de aberraciones cromosómicas.

Evaluación del periodo del ciclo celular promedio del poblado celular de Allium cepa L.: Se evaluó tomando cafeína $0,1 \%$ por una hora de iniciado los tratamientos. Se estableció previamente los índices interfásicos y mitótico promedio como se exhibe en la tabla 1 presentándose los promedios de la interfase y las fases de la mitosis (Gonzales, 2013). Dichos 
valores permitieron computar a su vez, los promedios en horas.

Al saber los tiempos del ciclo celular de Allium cepa L.; el grupo experimental A fue el testigo. En el grupo experimental $\mathrm{B}$, las raicillas de cebolla, fueron sometidas a la acción de la penicilina benzatínica $1 \%$ a partir de la cuarta hasta la sexta hora en la etapa de síntesis ("S") del periodo inicial de interfase a fin de estimular la formación de trastornos cromosómicos. En el grupo experimental C se supeditaron a la acción combinada de la penicilina benzatínica $1 \%$ y del extracto (tónico INTI) con el fin de lograr la reparación cromosómica esperada, para tal acción se usó un diseño experimental (Gonzáles y Chotón, 2019).

Repeticiones: Se trabajó con 6 bulbos de cebolla para cada uno de los 5 grupos.

Análisis estadístico e interpretación de datos: Obtenidos los resultados genuinos se aplicó la prueba de ANVA, utilizándose el programa estadístico SSPS versión 21,0. Los datos obtenidos fueron sujetados a las medidas de tendencia central (media) y de dispersión (varianza, error estándar), previo cambio arco-sen de los porcentajes originales.

\section{RESULTADOS}

Tabla1. Índices y tiempos promedio de los periodos y fases del ciclo celular del poblado celular de Allium cepa $\mathrm{L}$.

\begin{tabular}{|c|c|c|c|c|c|c|}
\hline \multirow{2}{*}{ Indicadores } & \multicolumn{2}{|c|}{ Ciclo celular } & \multicolumn{4}{|c|}{ Fases de la mitosis } \\
\hline & Interfase & Mitosis & Profase & Anafase & Anafase & Telofase \\
\hline Indices promedio & Interfas & 14,6 & 44,6 & 15,4 & 9,2 & 30,8 \\
\hline Varianza & 3,1 & 2,8 & 3,2 & 1,4 & 1,8 & 2,7 \\
\hline Error estándar & 0,7 & 0,75 & 0,80 & 0,53 & 0,60 & 0,73 \\
\hline Duración promedio (HS) & 11,2 & 2,0 & 0,89 & 0,32 & 0,18 & 0,61 \\
\hline
\end{tabular}

En la tabla 1 consideramos la media aritmética de los índices de las perduraciones del ciclo celular y de las fases de la mitosis de A. cepa; a partir del 14,6\% de células mitósicas descubiertas se distinguieron los índices de cada fase mitótica siendo profase y anafase la más y menos numerosa $(44,6 \%$ y $9,2 \%)$, respectivamente.

Tabla 2: Proporción promedio de los tipos de trastornos cromosómicos descubiertos en tejidos de formación de Allium cepa $\mathrm{L}$. tratados con penicilina benzatínica $1 \%$

\begin{tabular}{lcccc}
\hline & $\begin{array}{c}\text { Puentes } \\
\text { Cromosómicos }\end{array}$ & $\begin{array}{c}\text { Fragmentaciones } \\
\text { cromosómicas }\end{array}$ & $\begin{array}{c}\text { Otras trastomos } \\
\text { cromosómicos }\end{array}$ & $\begin{array}{c}\text { Sin } \\
\text { trastomos }\end{array}$ \\
\hline CELULAS ANAFASICAS & 11,3 & 16,7 & 38,3 & 33,4 \\
VARIANZA & 1,6 & 3,5 & 4,1 & 1,3 \\
ERROR STANDARD & 0,03 & 0.71 & 0.92 & 0.58 \\
\hline
\end{tabular}

Respecto a la tabla 2 advertimos las proporciones de trastornos cromosómicos tipo: puentes cromosómicos $(11,3 \%)$, fragmentaciones cromosómicas $(16,7 \%)$, otros trastornos cromosómicos $(38,3 \%)$ y sin trastornos $(33,4 \%)$ encontradas en células mitósicas durante el proceso celular de A. cepa con sus escalas (medidas) centrales y de dispersión.

Tabla 3: Proporción promedio de los tipos de trastornos cromosómicos descubiertos en tejidos de formación de Allium cepa. L. tratados con penicilina benzatínica $1 \%$ más el tónico INTI.

\begin{tabular}{lcrcr}
\hline $\begin{array}{c}\text { Puentes } \\
\text { Cromosómicos }\end{array}$ & $\begin{array}{r}\text { Fragmentaciones } \\
\text { cromosómicas }\end{array}$ & $\begin{array}{c}\text { Otros trastomos } \\
\text { cromosómicos }\end{array}$ & $\begin{array}{c}\text { Sin } \\
\text { trastomos }\end{array}$ \\
\hline CÉLULAS ANAFASICAS & $* 2,1$ & $* 7,2$ & $* 10,4$ & $* 80,3$ \\
VARIANZA & 0,3 & 0,9 & 1,8 & 0,6 \\
ERROR STANDARD & 0,09 & 0,04 & 0,43 & 0,5 \\
\hline
\end{tabular}

Respecto a la tabla 3 exponemos los promedios de trastornos cromosómicos tipo: puentes cromosómicos $(2,1 \%)$, fragmentaciones cromosómicas $(7,2 \%)$, otros trastornos cromosómicos $(10,4 \%)$ y sin trastornos $(80,3 \%)$ encontradas en células mitóticas durante el proceso celular de A. cepa con sus parámetros estadísticos descritos, los cuales nos demuestran el efecto reparador del extracto (tónico INTI) al hallar los porcentajes de daño cromosómico reducidos.

\section{CÉLULAS VIGOROSAS} EN INTERFASE

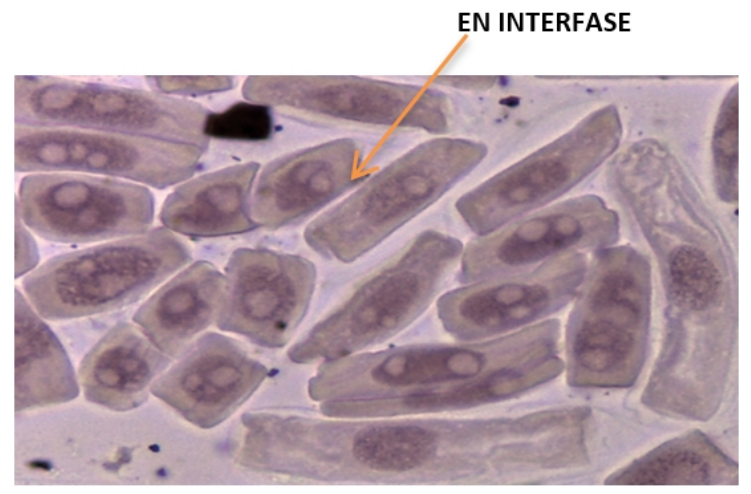

Fig.1: Tejido de Allium cepa L. manifestando células vigorosas en interfase por efecto del extracto (tónico INTI) (1000X).

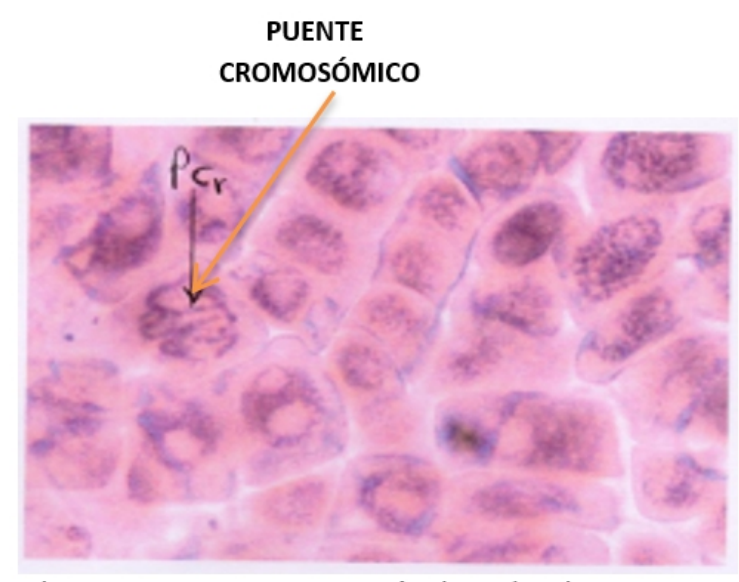

Fig.2: Trastorno cromosómico de tipo puente en Allium cepa L. ocasionada por la penicilina benzatínica $1 \%(1000 \mathrm{X})$. 


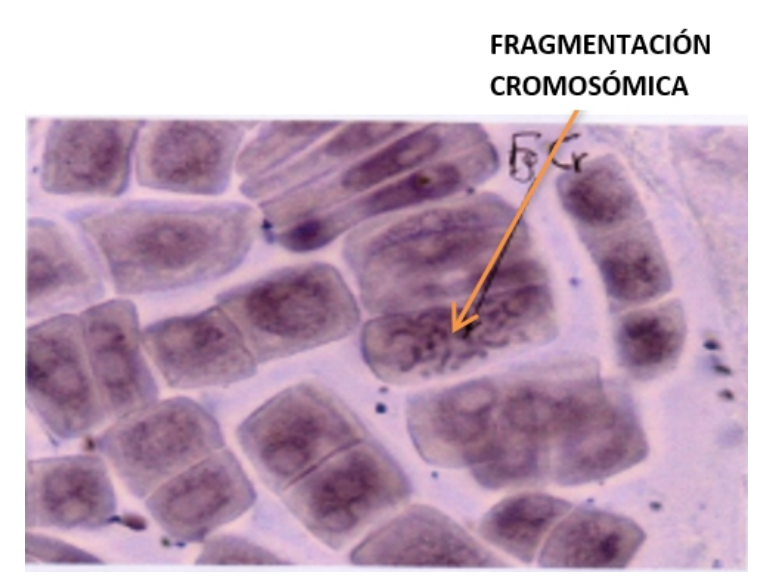

Fig.3: Trastorno cromosómico de tipo fragmentario en Allium cepa L. originado por la penicilina benzatínica $1 \%(1000 \mathrm{X})$.

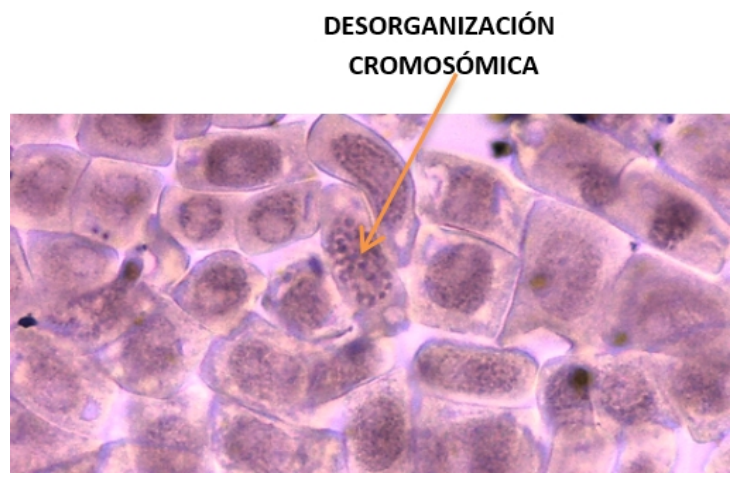

Fig.4: Trastorno cromosómico de tipo desorganización cromosómica en Allium cepa L. inducido por penicilina benzatínica $1 \%(1000 \mathrm{X})$.

\section{DISCUSIÓN}

En la tabla 1 se evaluó los valores del índice del ciclo celular y de las etapas mitóticas de los tejidos de A. сера que ya fueron analizados y calculados (Gonzáles y Diaz, 2016); encontrando el tiempo de 11,2 hs. para interfase y de 2,0 hs. para mitosis, (Gonzáles, 2019). En la subetapa final de síntesis de ADN se incitó correctamente las alteraciones cromosómicas (Stent y Calendar, 2014; Alberts et al., 2014).

Esta reducción del porcentaje en el reordenamiento cromosómico posiblemente se debió al efecto de los componentes del extracto (aminoácidos y vitaminas) los cuales habrían estimulado los mecanismos de reparación celular de los tejidos formadores de A. cepa L. y que habrían sido dañados por efecto de la penicilina. Los frutos de este estudio comparado con lo hallado por Gonzales (2013 y 2015) quien utilizó el gel de Aloe vera L. "sábila" y el infuso de Piper aduncum L. "matico" como agentes bioreparadores de material cromosómico dañado inducidos por antibióticos (amoxicilina 1\% y ciprofloxacina 1\%) respecto a las células que no exhibieron trastornos cromosómicos $(95,9 \%$ y 96,8\%) se descubrió que la dosis empleada del extracto ensayado no fue superior $(88,1 \%)$ en tejidos embrionarios de Allium cepa $\mathrm{L}$.

Se indica en la tabla 2 que los puentes cromosómicos fueron inducidos con penicilina benzatínica $1 \%$, en un $11,3 \%$ de células anafásicas de A. cepa L.; el alto poder mutagénico del antibiótico ensayado que comparado con el efecto de otras sustancias químicas mutagénicas como el 2,4-D (ácido diclorofenoxiacético) (Gonzales, 2004) habrían generado estas aberraciones cromosómicas (figura 2). Los cromosomas fueron rotos en su doble estructura y podría entenderse como la acción de la penicilina en el preciso momento en que las células se replicaban y/o reparaban. Es notable (tabla 3) la eficiencia del extracto (tónico INTI) que al haber suministrado los aminoácidos y vitaminas esenciales a las raicillas de A. cepa conjuntamente con el antibiótico logró reducir el daño $(2,1 \%$ de puente cromosómico).

El poder destructor cromosómico de la penicilina benzatínica $1 \%$ se observa en la tabla 3 donde la fragmentación se estimuló en un $16,7 \%$ de células profásicas de A. cepa L.; puesto que rompió muchos cromosomas tal como se demuestra en la figura 4. Mientras que los ápices de A. cepa que recibieron penicilina benzatínica $1 \%$ más el tónico INTI el nivel de daño cromosómico disminuyó porcentualmente $(7,2 \%)$.

Al analizar el daño cromosómico en profase, esto advierte que el efecto de la penicilina benzatínica $1 \%$ estaría evitando la separación de la carioteca, paso decisivo que resalta el término de la profase y su traslado a la metafase (Alberts et al., 2014).

Además la tabla 3 nos señala la proporción de 38,3\% de otras aberraciones cromosómicas (reorganización cromosómica con múltiples fragmentaciones) tal como lo examinamos en la figura 4 y que con la aplicación del extracto este porcentaje se vio reducido al hallar el $10,4 \%$ de daño del material nuclear. Estos hallazgos comparados con lo encontrado por Beltrán y Gonza (2016) quienes usando agua contaminada con metales pesados del río Moche (Trujillo) hallaron en células de Vicia faba L. aberraciones cromosómicas similares en un alto porcentaje. Se puede avizorar que los microtúbulos quienes orientan a los cromosomas desde la metafase y anafase a tomar una ubicación adecuada para la posterior división celular, fueron desorganizados por la sustancia química en estudio; por lo tanto la maquinaria mitótica celular fue desbaratada por la penicilina (López y Valentine, 2015; Vicente y Wordeman, 2015).

Estos hallazgos sobre fragmentaciones cromosómicas concuerdan con lo encontrado por 
otros estudiosos (Restrepo et al., 2012; Mohammed y Ghasssan, 2014;) quienes utilizaron glifosato (herbicida) y moléculas aminoquinolínicas como inductores de aberraciones cromosómicas sobre raicillas de Vicia faba L. y Allium cepa L. respectivamente.

En el año 2007 Gonzales indujo alteraciones cromosómicas en Allium cepa L. en porcentajes de $21.8 \%, 27.9 \%$ y $8.8 \%$ utilizando como agente citotóxico al ibuprofeno. Posteriormente el mismo autor logró revertir el daño cromosómico ocasionado por el ibuprofeno al utilizar como agente reparador de dicho daño al extracto de Aloe vera $\mathrm{L}$. logrando reducir en $0.4 \%, 3.6 \%$ y $5.8 \%$ demostrando que es posible en el laboratorio contrarrestar los efectos indeseables producidos por ciertos de agentes químicos que propician la contaminación humana y ponen en riesgo cada día la vida del ser humano.

El gran aumento de células interfásicas saludables de A. cepa L. $(80,3 \%)$ como se indica en la tabla 3 y se contempla en la figura 1 podría deberse a dos factores decisivos 1) a la fuerte estimulación y activación de la reparación tisular ejercida por el extracto; 2 ) a la intervención de agentes externos (penicilina benzatínica $1 \%$, cambio de $\mathrm{pH}$ y de temperatura y otros factores) del medio ambiente a las cuales fueron sometidas los tejidos de $A$. cepa $\mathrm{L}$. que hicieron que sus células en el momento de la división se detuvieran e ingresaran a una etapa de reposo (etapa Go) siendo una forma de enfrentar a un medio ambiente desfavorable (Salomon et al., 2012); en esto concordarnos con Gonzales (2013) quien sometió a tejidos de $A$. cepa $\mathrm{L}$. ante el efecto de la amoxicilina llevándolo a similares conclusiones. La penicilina benzatínica $1 \%$ habría ejercido un fuerte efecto citostático activando y desactivando muchas sustancias biológicas durante el ciclo celular (figura 2). Esto concuerda con el trabajo realizado por Beltrán (2013) quien usando el metamidofos (plaguicida) como agente citotóxico observó un fuerte efecto citostático, al detenerse el ciclo celular (Go) de Allium cepa L.

La citotoxicidad también tiene su acción sobre la fase nucleolar alterando su formación estructural como su fisiología celular (Silva et al., 2016; Huaman et al., 2014). El estudio del nucléolo ha sido tomado como un indicativo en diversos trastornos patológicos en seres humanos. Recientes investigaciones familiarizan la alteración del nucléolo con formas cancerígenas tales como melanomas y carcinomas, los cuales representan índices biológicos de transformación cancerígena (Beltrán y Beltrán, 2016; Bernstein y Bernstein, 2014).

El extracto enriquecido con proteínas y vitaminas (tónico INTI) posee un potente efecto reparador al reducir proporcionalmente los daños cromosómicos descubiertas en tejidos formadores de Allium cepa $L$. "cebolla" tratadas con penicilina benzatínica $1 \%$.

En las condiciones de laboratorio empleadas a una dosis de $10 \mathrm{~mL}$ del extracto utilizado se comprobó el notable efecto historeparador.

\section{CONCLUSIONES}

En las condiciones de laboratorio empleadas a una dosis de $10 \mathrm{~mL}$ del extracto utilizado se comprobó el notable efecto historeparador.

Los tejidos formadores de $A$. cepa $\mathrm{L}$. tratados con penicilina benzatínica $1 \%$ presentaron trastornos cromosómicos tipo: puentes, fragmentaciones y otras aberraciones y sin aberraciones en porcentajes de: $11,3 \%, 16,7 \%, 38,3 \%$ y $33,4 \%$ mientras que el grupo que recibió el antibiótico más el extracto presentó: puentes $(2,1 \%)$, fragmentaciones $(7,2 \%)$, y otras aberraciones $(10,4 \%)$ y $\sin$ aberraciones $(80,3 \%)$ respectivamente.

El extracto proteico y vitamínico (tónico INTI) posee un potente efecto reparador al reducir proporcionalmente las aberraciones cromosómicas descubiertas en radículas de Allium cepa tratadas con penicilina benzatínica $1 \%$.

\section{REFERENCIAS BIBLIOGRÁFICAS}

Alberts, B.; Johnson, A.; Lewis, J.; Morgan, D.; Raff, M.; Roberts, K.; Walter, P. (2014). Biología Molecular de la célula. 6ta. ed. New York. Garland Science.

Beltrán, R. (2013). Acción genocontaminante del agroquímico metamidofos y del saborizante glutamato monosódico en el ciclo nucleolar de Allium cepa L. Conocimiento para el desarrollo. 2(1):109-116.

Beltrán, R.; Gonza, K. (2016). Citotoxicidad y genotoxicidad de las aguas de los ríos Jequetepeque y Moche mediante el bioindicador ambiental Vicia faba $L$. "haba". Rev. Scientia Agropecuaria 8 (3): 203-213.

Beltrán, R.; Beltrán, P. (2016). Regulación del ciclo celular $(C C)$ de Vicia faba L. por el extracto alcohólico de Annona cherimola Mill "chirimoya". Rev. Scientia Agropecuaria 7 (3): $245-251$.

Bernstein, R.; S. Bernstein. (2014). Biología. Edit. Mac Graw Hill Inter. México.

Causil, L.; Coronado, J.; Verbel, L.; Veja, J.; Donado, 
E.; Pacheco, G. (2017). Efecto citotóxico del hipoclorito de sodio ( $\mathrm{NaClO}$ ), en células ápicales de raíces de cebolla (Allium cepa). Revista Colombiana De Ciencias Hortícolas 11(1): 97-104.

Croce, C; Klein, V. (2013). Translocaciones cromosómicas y cáncer humano. En Cairos Eds. El cáncer pp. 117 123. Prensa Científica. España.

Gonzáles, L. (2004). Procesos de transformación celular en Alliun cepa L. Chachapoyas. UNTRM. Chachapoyas. Perú.

Gonzáles, L. (2007). Efecto reparador de A. vera "sábila" en tejidos mitóticos de A. cepa con daño cromosómico por efecto del ibuprofeno. UNTRM. Chachapoyas- Perú.

Gonzáles, L. (2015). Efecto reparador de Piper aduncum L. "matico" en tejidos embrionarios de Allium cepa L. "cebolla" con lesiones cromosómicas inducido por ciprofloxacina. Chachapoyas. UNTRM. Chachapoyas- Perú.

Gonzales, L.; Díaz, C. (2016). Efecto citoreparador de Aloe vera L. "sábila" en tejidos embrionarios de Allium cepa L. "cebolla" con daño cromosómico inducido por amoxicilina. Acc Cietna 2(2), 1-10.

Gonzales, L.; Chotón, M. (2019). Efecto bioprotector de Croton lechleri L. frente a la toxicidad del meloxicam en Allium cepa $L$. Chachapoyas. Trabajo de investigación. UNTRM. Chachapoyas- Perú.

Gonzales, L.; Chotón, M.; Chico, J. (2020). Efecto citotóxico y genotóxico de la dexametasona e ibuprofeno en radículas de Allium cepa $L$. “cebolla”. Manglar 17(3): 227-232.

Haq, I.; Kumar, S.; Raj, A.; Lohani, M.; Satyanarayana, G. (2017). Genotoxicity assessment of pulp and paper mill effluent before and after bacterial degradation using Allium cepa test. Chemosphere 169: 642-650.

Huamán, F. D.; Toscano, E.; M.; Acosta, O.; Rojas, D. E.; Inocente, A.; Garrido, D.; GuevaraFujita, M. (2014). Estudio genotóxico de una bebida experimental de quinua, kiwicha y kañiwa. Rev. Per. Biol. 21 (3):25258.

Katzung, B.; Trevor, A. (2016). Farmacología Básica y Clínica. 13ava. ed. Mc Graw Hill

\section{S.A. México}

Kir, G.; Sarbay, B. C.; Gumus, E. (2016) Significance and outcome of nuclear anaplasia and mitotic index in prostatic adenocarcinomas. Urologic Oncologic. 34 (10): 430.e9-430.

Kuchy, A.H., A.A.Wani y A.N. Kamili. (2016). Cytogenetic effects of three commercially formulated pesticides on somatic and germ cells of Allium cepa. Environ. Sci. Pollut. Res. 23(7), 6895-6906.

López, B. J.; Valentine, M. (2015). Molecular control of stress transmission in the microtubule cytoskeleton. Biochimica et Biophysica Acta 1853:3015-3024.

Malca, J.; Incio, M.; Ñique, C.; Rodríguez, L.; Manchay, R.; Malca, J. (2020). Evaluación del indice mitótico de meristemos radiculares de Allium cepa "cebolla" expuestos a diferentes concentraciones de metotrexato. Medicina Naturista. 14(2).

Mamani, D. (2017). Efecto de cuatro concentraciones del extracto acuoso de hojas y flores de Brassica rapa l. en el ciclo celular de meristemos radiculares de Allium sativum L. Tesis Bach. UNA. Puno. Perú.

Mohammed, A. A.; Ghassan, M. (2014). Mutagenic effects of glyphosate (IPA) herbicides using the Vicia faba L. Root tip. World Journal of Biology and Biological Sciences 2 (2):044048.

Morón, F.; Levi, M. (2014). Plantas medicinales y medicamentos herbarios. Farmacología G. La Habana: C. Médicas; p. 195-205.

Oram, R. (2013). Biología. Edit. Mac Graw Hill Interamericana. México.

Rang, H.; Flower, R.; Ritter, M.; Anderson, G. (2016). Farmacología. Studentconsult. Edit. Elsevier.

Restrepo, R.; Reyes, D.; Ortiz, M.; Rojas, F.; Kouznetsov, V. (2012). Aberraciones cromosomales en bulbos de cebolla Allium cepa inducidas por moléculas híbridas 4 aminoquinolínicas. Universitas Scientarium 17(3): 253-261.R

Salomon, E.; L. Berg.; D, Martin. (2012). Biología. 5ta. Edición. Edit. Mac Graw Hill Interamericana. México.

Silva, N. M.; Resende, M. R.; Morales, D. A.; 
Umbuzeiro, G.; Gomez, M. F. (2016). In vitro mutagenicity assay (Ames test) and phytochemical characterization of sedes oil Helienthus annuus Linné. Toxicology reports 3:733-739.

Stent, G.; Calendar, R. (2014). Genética molecular. Ed.Omega S.A. Barcelona.

Vicente, J. J.; Wordeman, L. (2015). Mitosis, microtubule dynamics and the evolution of kinesis. Experimental Cell Research 334: 61-69. 